

\title{
SIMULACIÓN MEDIANTE CDF DE UNA CHIMENEA SOLAR INCLINADA SOBRE LA CUBIERTA
}

\section{CFD SIMULATION OF AN INCLINED ROOF SOLAR CHIMNEY}

\author{
SANDRA RODRÍGUEZ-TREJO \\ Magíster en Diseño Bioclimático \\ Estudiante doctoral, Posgrado en Diseño Bioclimático, \\ Dpto. de Medio Ambiente \\ Universidad Autónoma Metropolitana Azcapotzalco \\ Ciudad de México, México \\ https://orcid.org/0000-0002-6139-4985 \\ sandraelisar@gmail.com
}

\author{
VÍCTOR FUENTES-FREIXANET \\ Doctorado en Diseño, Arquitectura Bioclimática \\ Académico Investigador, \\ Universidad Autónoma Metropolitana Azcapotzalco \\ Ciudad de México, México \\ https://orcid.org/0000-0002-7426-2391 \\ ffva@azc.uam.mx
}

RESUMEN

En los últimos años se ha elevado el consumo energético derivado del uso de aparatos eléctricos para promover el movimiento de aire en regiones con climas cálidos, lo que ha traído como consecuencia un impacto negativo en el ambiente. En este trabajo se evalúa el desempeño de una chimenea solar utilizada para inducir la ventilación natural en un espacio cerrado, bajo las condiciones del clima cálido mexicano. Para tal efecto, se desarrollaron simulaciones CFD empleando el modelo de turbulencia RNG k- e y el modelo de radiación DO, y considerando únicamente los fenómenos de convección natural. El desempeño de la chimenea solar se evaluó comparando los resultados de las simulaciones con mediciones experimentales; análisis que reveló una buena concordancia. Se obtuvieron temperaturas de hasta $46.5^{\circ} \mathrm{C}$ en el aire dentro de la chimenea y de $77.1^{\circ} \mathrm{C}$ en la placa de absorción; resultados que permiten verificar la influencia del fenómeno de descarga de calor por flotación natural del aire en la chimenea.

Palabras clave

energía solar, ventilación, simulación CFD

\begin{abstract}
In recent years, energy consumption from electrical devices to foster air movement in regions with warm climates

has risen, with the resulting negative impact on the environment. The purpose of this paper is to evaluate the performance of a solar chimney used to induce natural ventilation in a closed space, under the weather conditions of the hot humid Mexican climate. For this purpose, CFD simulations were run using the RNG k-e turbulence model and the DO radiation model, considering only natural convection phenomena. The solar chimney performance was evaluated, comparing the results of the simulations with experimental measurements, analysis which showed a good match. Temperatures of up to $46.5 \%{ }^{\circ} \mathrm{C}$ in the air within the chimney, and of $77.1^{\circ} \mathrm{C}$ on the absorption plate, were obtained, results that allow verifying the influence of the heat discharge phenomenon by the natural flotation of air in the chimney.
\end{abstract}




\section{INTRODUCCIÓN}

Cerca del $30 \%$ del consumo eléctrico del sector residencial de México se utiliza para lograr el confort térmico en zonas de clima cálido (González y Beele, 2016), proporción que sigue aumentando notablemente en la medida en que también lo hace el uso de equipos de climatización de aire en los últimos años. La cantidad de energía eléctrica que se emplea para climatización artificial en climas cálidos depende de varios factores, específicamente del diseño y los elementos de la envolvente del espacio, como también de los equipos que proveen los tres elementos que determinan el confort térmico: temperatura, humedad y velocidad del aire (de Buen, 2017).

Los resultados de la Encuesta Nacional sobre Consumo de Energéticos en Viviendas Particulares (Instituto Nacional de Estadística y Geografía [INEGI], 2018) muestran que en el país se contabilizan 14.6 millones de ventiladores. Los de uso más común en las viviendas, aquellos conocidos como de techo y pedestal, registran 11.5 millones, presentando además un uso preponderante: en el $34.4 \%$ de las viviendas se utilizan entre 5 y 9 horas al día.

Estos datos proporcionan una perspectiva general del consumo energético que origina el uso de dispositivos para promover el movimiento de aire en climas cálidos, y su consiguiente impacto económico y ambiental. De ello se deriva la importancia de la implementación de estrategias pasivas de climatización para favorecer la ventilación natural y proveer confort térmico.

La ventilación natural en los edificios es originada por dos causas: por presiones debidas al viento (ventilación cruzada) y por diferencia de temperatura, por lo tanto, de densidad del aire, entre el exterior y el interior (efecto Stack). En climas cálido-húmedos, la ventilación por efecto Stack es ineficiente ya que la diferencia de temperatura entre el interior y el exterior de los edificios ventilados naturalmente, es poca. En este contexto, las chimeneas solares representan una alternativa eficiente para promover la ventilación natural, gracias a su capacidad de aprovechamiento del recurso solar para calentar una parte de la edificación propiciando la extracción de aire cálido por efecto convectivo.

Una chimenea solar es básicamente un calefactor de aire solar que se incrusta vertical u horizontalmente a una edificación como parte de una pared o techo, y cuya clasificación puede variar según diferentes configuraciones o funciones (Lal, Kaushik y Bhargav, 2013). Se pueden encontrar distintas variaciones en el diseño de las chimeneas solares influenciadas por una serie de factores, tales como la ubicación, el clima, la orientación, el tamaño del espacio a ventilar y las ganancias de calor internas. Sin embargo, los elementos básicos que las conforman como el colector solar, la cubierta transparente y las aberturas de entrada y salida forman parte de cada diseño (Khanal y Lei, 2011).

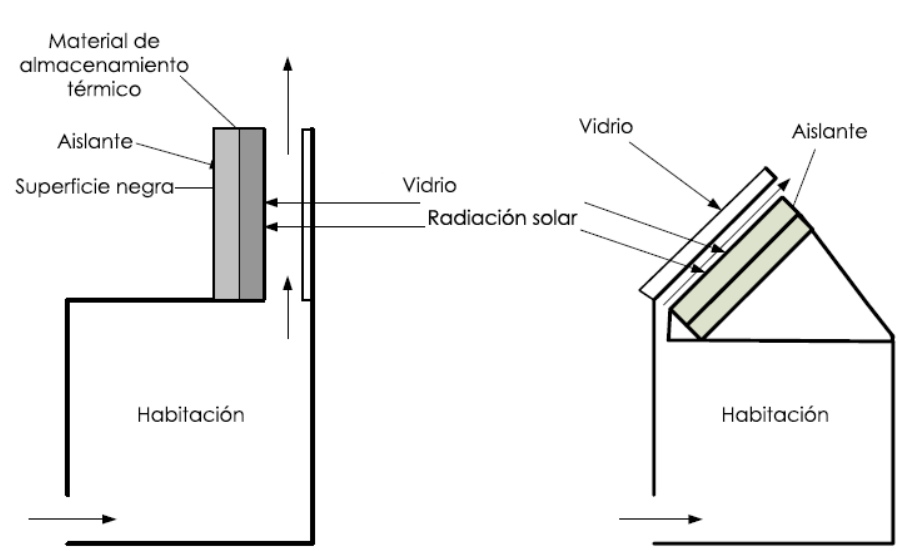

Figura 1. Configuraciones de chimenea solar. Fuente: Harris y Helwig (2007, p. 136).

\section{CARACTERÍSTICAS Y FUNCIONAMIENTO DE LAS CHIMENEAS SOLARES EN CUBIERTA}

Para promover la ventilación natural, las chimeneas solares inclinadas sobre la cubierta o el techo funcionan con los mismos principios que las chimeneas verticales, es decir, se asisten del efecto de succión en las aberturas situadas en la parte baja de la chimenea y del aire caliente que se extrae a través de las aberturas superiores del canal (Figura 1). Generalmente, las chimeneas solares se componen de una cubierta transparente y una placa de absorción o de almacenamiento térmico separadas por una cavidad o canal de aire; en la parte trasera de la placa se dispone una capa de aislamiento para evitar pérdidas de calor.

En la mayoría de los casos, la cubierta transparente de la chimenea solar es de vidrio, que es un material transparente a la radiación de onda corta; una parte de la radiación solar incidente en la superficie del vidrio se transmite directamente al ambiente interno, otra se refleja hacia el exterior y una tercera parte es absorbida por el material. Como el vidrio tiene baja absorción y está expuesto a los intercambios de calor por convección en sus dos caras, su temperatura superficial es siempre cercana a la del aire con el que está en contacto.

La placa de absorción es comúnmente de material metálico con altos valores de emisividad y absortancia, y se calienta por absorción de la porción de radiación solar transmitida por el vidrio. Una parte de este calor puede perderse por conducción hacia el lado externo de la placa, donde hay aislamiento térmico, y otra parte es cedida al aire en la cavidad por convección térmica y al vidrio, por radiación infrarroja. De esta forma, con el aumento de temperatura del aire presente en la cavidad de la chimenea, que es calentado por la placa absorbente y el vidrio, se genera el movimiento ascendente del aire hacia la abertura superior.

La estrategia de integrar las chimeneas solares a lo largo de la pendiente del techo o cubierta de las edificaciones permite obtener grandes áreas para colectar irradiancia 
solar y aprovecharla cuando el sol está a mayor altitud. Algunas desventajas de estos sistemas son que la altura efectiva está restringida por el ángulo de inclinación del techo y la posibilidad de mayores pérdidas de presión debido a los cambios de dirección adicionales en el recorrido del aire (Harris y Helwig, 2007).

\section{INVESTIGACIONES PREVIAS}

A partir de los años 90 se ha realizado una cantidad significativa de investigaciones, a través de métodos experimentales, analíticos y computacionales, enfocada sobre todo en encontrar las soluciones de diseño óptimas para mejorar la ventilación natural utilizando chimeneas solares. De acuerdo con Shi et al. (2018), el desempeño de estos sistemas se basa principalmente en ocho factores: ángulo de inclinación, ancho de cavidad, altura, relación dimensional entre altura/cavidad, área de abertura de entrada, área de abertura de salida, relación entrada/ salida y radiación solar. Zhang, Tan, Yang y Zhang (2016) mencionan, además, las características térmicas del material de absorción de la radiación solar.

El ángulo de inclinación de las chimeneas solares es uno de los parámetros más analizados. Varias investigaciones reportan que una inclinación de $45^{\circ}$ es óptima para obtener un máximo flujo de aire (Chen et al., 2003; J. Mathur, Bansal, S. Mathur, Jain y Anupma, 2006; Bassiouny y Korah, 2009; Saifi, Settou, Dokkar, Negrou y Chennouf, 2012; Jianliu y Weihua, 2013; Al-Kayiem, Sreejaya y Gilani, 2014; Saleem, Bady, Ookawara y Abdel-Rahm, 2016; Dhahri y Aouinet, 2020; Kong, Niu y Lei, 2020), inclinación que genera pocas pérdidas de presión, pues representa un equilibrio entre la presión stack y la transferencia de calor por convección (Yusoff, Salleh, Adam, Sapian y Sulaiman, 2010; Mahdavinejad, Fakhari y Alipoor, 2013).

Las recomendaciones para el dimensionamiento del espesor de cavidad varían entre los $0.10 \mathrm{~m}$ a los 0.35 $m$. Diversos estudios concuerdan en que el flujo de aire disminuye con el aumento del espesor de la cavidad por la presencia de flujo inverso. En investigaciones realizadas por Shi et al. (2016), Saifi et al. (2012), Yusoff et al. (2010) y Zhai, Dai y Wang (2005) no se registran flujos inversos en cavidades de $0.20 \mathrm{~m}$. Bouchair (1994), Li, Jones, Zhao y Wang (2004) y Liping y Angui (2004), por su parte, reportan como óptima la relación 1:10 entre el ancho de cavidad y la altura efectiva de la chimenea.

Los trabajos realizados sobre el aumento en la longitud de la chimenea solar han mostrado menor impacto de esta característica geométrica en el desempeño de las chimeneas en comparación con el aumento en el espesor de la cavidad. El rango de longitudes de chimenea analizadas en investigaciones previas va de los $0.40 \mathrm{~m}$ hasta los $3.00 \mathrm{~m}$. En tal sentido, Neves (2012) sostiene que después de los $1.50 \mathrm{~m}$ las variaciones en la longitud resultan en incrementos progresivamente menores en el flujo de aire.
En general, el rango de las variables de configuración analizadas en los estudios previos es limitado: se centran principalmente en la anchura de la cavidad y el ángulo de inclinación. Los valores óptimos sugeridos no son aplicables a todas las configuraciones de chimenea solar ya que presentan relaciones de dependencia recíproca entre dos o más variables, por lo que es necesario llevar a cabo más investigaciones a fin de evaluar su desempeño bajo diferentes contextos.

\section{DINÁMICA DE FLUIDOS COMPUTACIONAL}

La Dinámica de Fluidos Computacional (CFD, por sus siglas en inglés) es una rama de la mecánica de fluidos que utiliza estructuras de datos y métodos numéricos para resolver y analizar las ecuaciones que gobiernan el flujo de fluidos y permite conocer cuantitativa y cualitativamente el comportamiento de los mismos.

La simulación CFD es uno de los métodos más importantes para el estudio de la ventilación natural y puede ser empleada para predecir el flujo de aire y la transferencia de calor al interior y exterior de las edificaciones. Los modelos CFD son capaces de proporcionar una distribución espacial detallada de la temperatura, presión, velocidad y turbulencia del aire (Sánchez, 2017).

Una de las técnicas numéricas más utilizadas para discretizar las ecuaciones que describen el movimiento de los fluidos (Navier-Stokes) es el método de los volúmenes finitos, cuyo punto de partida es la descomposición del dominio a analizar en un número finito de volúmenes de control contiguos, donde las variables son calculadas en los nodos localizados en el centroide de cada volumen de control. Los volúmenes de control y los nodos son definidos con una malla numérica, que es esencialmente una representación discreta del dominio en el cual el problema es resuelto.

En el siguiente apartado se presenta la descripción del modelo de simulación CFD utilizado en esta investigación para analizar el flujo de aire a través de una chimenea solar. Cabe mencionar que las condiciones de borde fueron determinadas con base en resultados experimentales obtenidos del monitoreo en campo de un modelo físico a escala real.

\section{METODOLOGÍA}

El trabajo aquí expuesto tiene un enfoque cuantitativo (se vale de herramientas matemáticas, estadísticas e informáticas). Se optó, asimismo, por efectuar un estudio experimental y un análisis numérico para la recolección y el análisis de datos, buscando relaciones causales entre las variables medidas. Se ha seguido el método científico analítico en cuanto al análisis de los resultados de la simulación y la comparación de resultados numéricos y experimentales. 

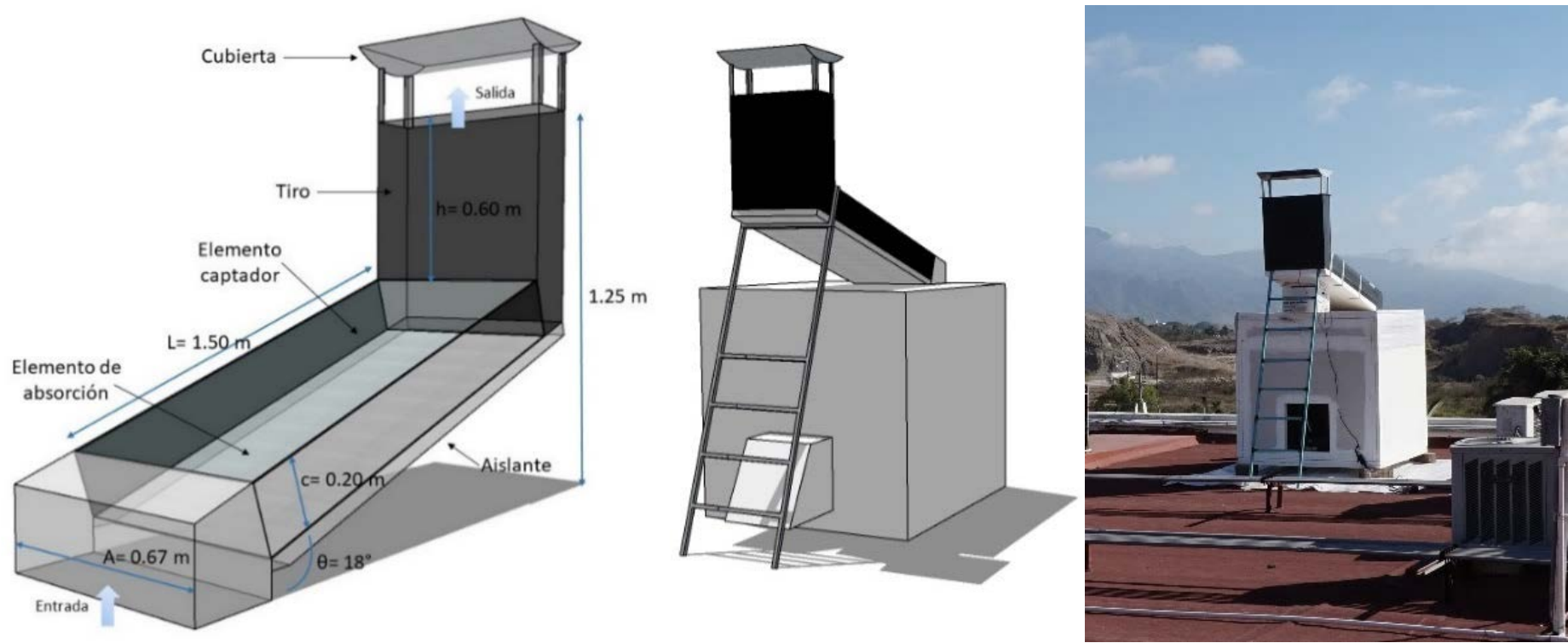

Figura 2. Características del modelo experimental. Fuente: Elaboración de autores.

El diseño de la investigación parte de dos fases fundamentales, la primera de ellas es el diseño de la chimenea solar a partir del análisis de la bibliografía existente y la determinación de los periodos de experimentación y las características constructivas del prototipo experimental. La segunda fase consistió en la aplicación de la técnica CFD para la simulación del sistema, de modo de validar, posteriormente, el modelo CFD utilizado a partir de los resultados experimentales y verificar si se ha encontrado una solución coherente para el problema.

\section{ESTUDIO EXPERIMENTAL}

El estudio experimental se realizó bajo las condiciones del clima de la ciudad de Puerto Vallarta, México, con coordenadas $20^{\circ} 42^{\prime}$ Norte y $105^{\circ} 13^{\prime}$ Oeste, a 10.0 msnm. Las variables climáticas, incluida la temperatura ambiente, humedad relativa, velocidad y dirección del viento e irradiancia global, fueron adquiridas de una estación meteorológica automática.

Se diseñó la configuración geométrica de la chimenea solar a analizar, estableciendo una inclinación de $18^{\circ}$ hacia el Sur para obtener adecuadas condiciones de irradiancia solar durante todo el año en la latitud del estudio. Se construyó un colector de lámina de acero galvanizado calibre 22 color negro mate, de $1.50 \mathrm{~m}$ de largo, $0.67 \mathrm{~m}$ de ancho y $0.20 \mathrm{~m}$ de espesor de cavidad. Para compensar la pérdida de presión ocasionada por el ángulo de inclinación bajo, se colocó una extensión vertical, llamado tiro o stack, de $0.60 \mathrm{~m}$ acoplado al colector.

El espacio a ventilar consistió en una cámara cúbica de paneles de yeso de $1.50 \mathrm{~m}$ por lado, en la cual se dispuso una abertura cuadrada de $0.50 \mathrm{~m}$ por lado, en la parte inferior de la cara vertical opuesta a la entrada del colector, a una distancia de $0.15 \mathrm{~m}$ de la base. Como elemento captador de la radiación solar, se usó una cubierta de vidrio claro simple de $4 \mathrm{~mm}$ de espesor y una lámina lisa de aluminio de $2.0 \mathrm{~mm}$ de espesor color negro mate, como elemento de absorción, aislada en su cara inferior con una placa de poliestireno de $5 \mathrm{~cm}$ de espesor. Las características del modelo experimental se presentan en la Figura 2.

Se colocaron sensores en la entrada y al interior de la cámara, al interior del colector, en la superficie de la placa de absorción y en la salida del tiro. Las variables a monitorear fueron:

- Velocidad del aire en la entrada de la cámara.

- Temperatura al interior de la cámara, temperatura de la placa.

- Temperatura del aire interior de la cavidad.

- Velocidad del aire en la entrada del colector.

- Velocidad en la salida del tiro.

Se registraron datos cada 5 minutos durante tres días en distintos meses a lo largo del año y, seguidamente, se calcularon promedios horarios y se realizaron análisis estadísticos para determinar los datos a utilizar en las simulaciones.

\section{SIMULACIÓN CFD}

La metodología utilizada para ejecutar simulaciones en la mayoría de los códigos CFD contiene tres etapas principales: pre-procesamiento, solución y post-procesamiento. A su vez, cada una de las etapas consta de las siguientes actividades: 

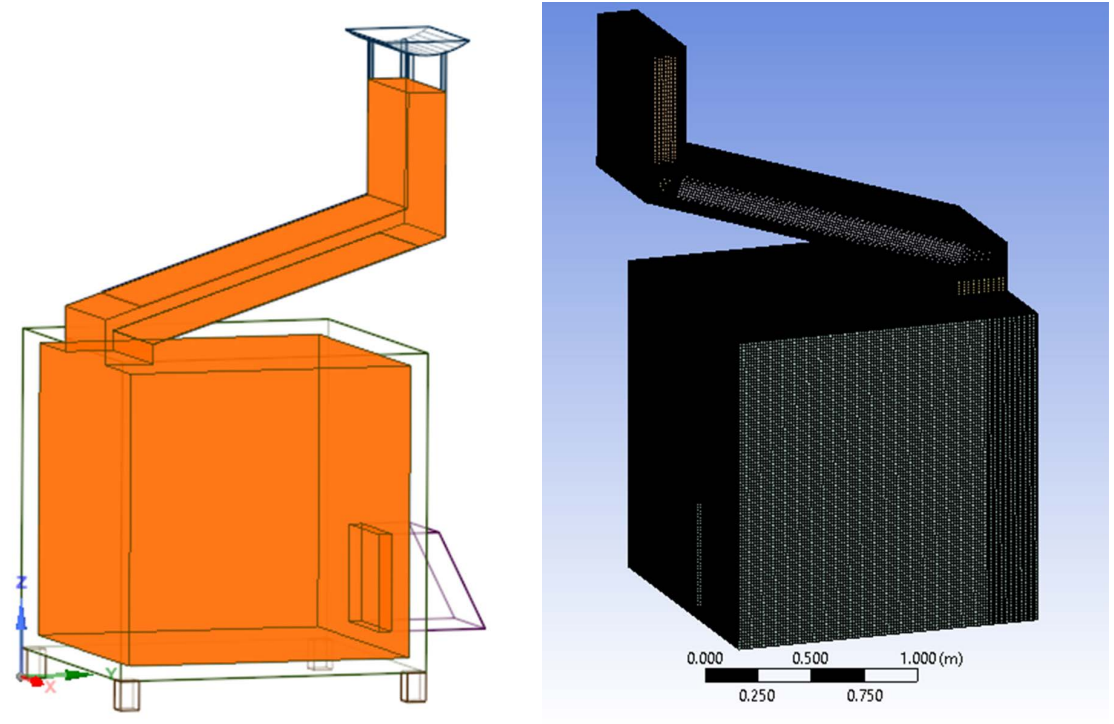

Figura 3. Modelo tridimensional y malla de análisis. Fuente: Elaboración de autores.

Pre-procesamiento:

- Elección del modelo de cálculo adecuado para resolver el problema específico y determinar el objetivo de la simulación.

- Definición de la geometría que representa el problema.

- Generación de una malla adecuada, considerando sus dimensiones y topología, así como la independencia de los resultados de la malla utilizada.

- Establecimiento de las propiedades de los fluidos.

Procesamiento o solución:

- Determinación de las condiciones de borde, tales como temperatura de las superficies, flujo de calor, entradas y salidas del fluido, fuentes y sumideros de calor, humedad, etc.

- Definición de los algoritmos de solución.

- Selección del modelo de turbulencia adecuado.

- Precisión de los parámetros numéricos como esquemas de diferenciación, factores de relajación, número de iteraciones, criterios de convergencia, etc.

\section{Post-procesamiento:}

- Obtención de datos, gráficos, vectores, líneas de flujo, contornos, etc. de las variables de interés.

- Validación del modelo comparando resultados con datos de experimentales u otros resultados numéricos.

Para el desarrollo del modelo y el análisis numérico se empleó el software comercial ANSYS Fluent. Con el objetivo de llevar a cabo las simulaciones de la chimenea solar, se seleccionó un modelo en tres dimensiones (Figura 3) con flujo en estado estacionario, contemplando únicamente los fenómenos de convección natural. En función de simular el flujo de aire a través del modelo, se ajustaron los parámetros a fin de obtener las mismas condiciones de la prueba experimental, considerando un flujo de aire turbulento, incompresible y estacionario.
Dada la configuración del modelo físico experimental de la chimenea solar, se optó por utilizar una malla estructurada conformada por hexaedros, cuyas dimensiones se configuraron acorde a las dimensiones de la chimenea procurando que su forma cumpliera los requisitos para que los cálculos numéricos fueran estables y exactos: alta calidad ortogonal y baja oblicuidad de los elementos. Se refinó la malla en las zonas de interés como la cubierta de vidrio, la placa de aluminio y la entrada y salida del aire. Con el proceso de mallado se obtuvieron 7 mallas, con un número de elementos entre 660711 y 2376 021, las cuales fueron configuradas y probadas para determinar el número de elementos adecuado para el modelo, permitiendo reducir costos computacionales sin perder calidad de la información. Se eligió para su análisis una malla conformada por 1633428 elementos.

Se activó la ecuación de Energía para habilitar el cálculo de los parámetros relacionados con la transferencia de calor y la variación de la densidad; también se activó el modelo de Viscosidad con el modelo de turbulencia RNG $k-e$, el cual es descrito como estable, dado que proporciona resultados razonablemente precisos para la mayoría de los flujos de aire interiores (Rodrigues, Frick, Bejat y Garrecht, 2021). Se eligió el modelo de radiación de Ordenadas Discretas (DO) que permite simular la radiación a través de medios semitransparentes, así como problemas de radiación con medios no participantes (como el aire) en los que la radiación influye indirectamente en el campo de flujo cambiando las condiciones de contorno en las superficies. De igual manera, se seleccionó el Método Semi-Implícito de Ecuaciones Ligadas para la Presión (SIMPLE) como algoritmo acoplamiento de la presión y velocidad.

Por último, se estableció como criterio de convergencia el valor de residuo de $10^{-6}$ para la ecuación de energía y de $10^{-3}$ para las demás variables de acuerdo con lo sugerido 


\begin{tabular}{|c|c|c|c|c|c|}
\hline \multirow{2}{*}{ Material } & Densidad $\rho$ & $\begin{array}{c}\text { Calor especifico } \\
\mathbf{C p}\end{array}$ & $\begin{array}{c}\text { Conductividad térmica } \\
\lambda\end{array}$ & Absortancia & Emisividad \\
\cline { 2 - 6 } & $\mathbf{k g} / \mathbf{m} \mathbf{3}$ & $\mathbf{J} / \mathbf{k g} \cdot \mathbf{K}$ & $\mathbf{W} / \mathbf{m} \cdot \mathbf{K}$ & $\alpha$ & $\mathbf{E}$ \\
\hline Vidrio & 2500 & 750 & 1.40 & 0.14 & 0.85 \\
\hline Aluminio & 2719 & 871 & 202.40 & 0.97 & 0.98 \\
\hline
\end{tabular}

Tabla 1. Propiedades termo-físicas y ópticas de los materiales. Fuente: Elaboración de autores.

en el manual de usuario de ANSYS.

El sistema opera a presión atmosférica a nivel del mar, por lo que se mantuvo el valor por defecto de $101325 \mathrm{~Pa}$. La temperatura operativa corresponde a la temperatura ambiente registrada durante los distintos periodos de experimentación. Para todos los casos a simular se consideró que el aire ingresa a la cámara de forma perpendicular a la abertura de entrada a una velocidad constante de $0.15 \mathrm{~m} / \mathrm{s}$ y se introdujeron valores de temperatura obtenidos durante la prueba experimental. La Tabla 1 exhibe las propiedades físicas de los materiales de los elementos que forman el modelo y que participan en el intercambio de energía.

\section{RESULTADOS Y DISCUSIÓN}

En el análisis de los resultados se utilizó el coeficiente de correlación lineal de Pearson (r) con el propósito de establecer similitudes entre las variables simuladas y los resultados experimentales. La Figura 4 muestra la dispersión de los datos de la temperatura de la placa obtenidos tanto en el monitoreo experimental como en las simulaciones. El coeficiente de correlación lineal entre las series de datos es igual a 0.93, lo que revela que existe una alta correlación positiva. La correlación entre la irradiancia y la temperatura de la placa obtenida en las simulaciones es de $r=0.97$, mientras que con los resultados experimentales es $r=0.91$.

La temperatura del aire dentro del colector de la chimenea está directamente relacionada con la temperatura de la placa, debido a la transferencia de calor por convección (Figura 5); el coeficiente de correlación entre estas variables en las mediciones experimentales es de $r=0.94$ y en las simulaciones es de $r=0.98$, lo que indica una alta correlación positiva.

En un análisis de dispersión de los datos de la temperatura del aire dentro del colector, se observa que la temperatura obtenida en las simulaciones es mayor a los datos experimentales cuando las condiciones de irradiancia son superiores a los $750 \mathrm{~W} / \mathrm{m}^{2}$ (Figura 6). El coeficiente de correlación entre ambas series de datos (experimentales y simulaciones) de temperatura del colector es de $r=0.87$.

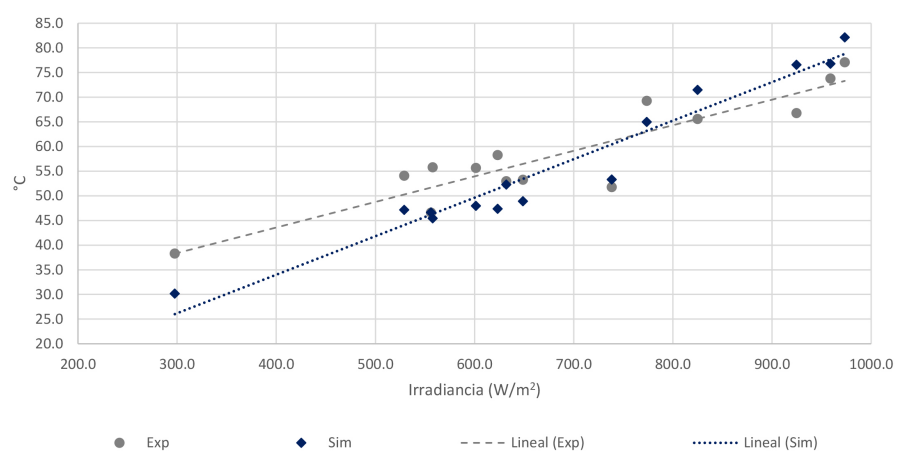

Figura 4. Temperatura de la placa de aluminio. Fuente: Elaboración de autores.

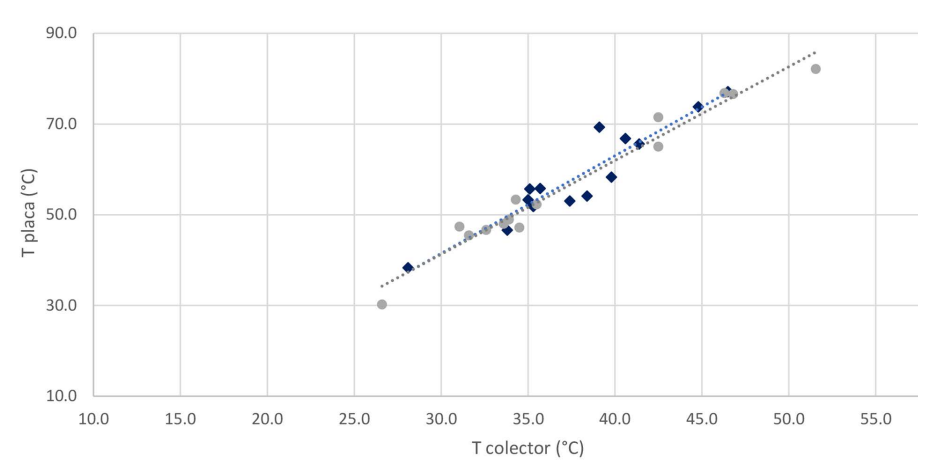

Figura 5. Correlación entre la temperatura del aire y la temperatura de la placa.

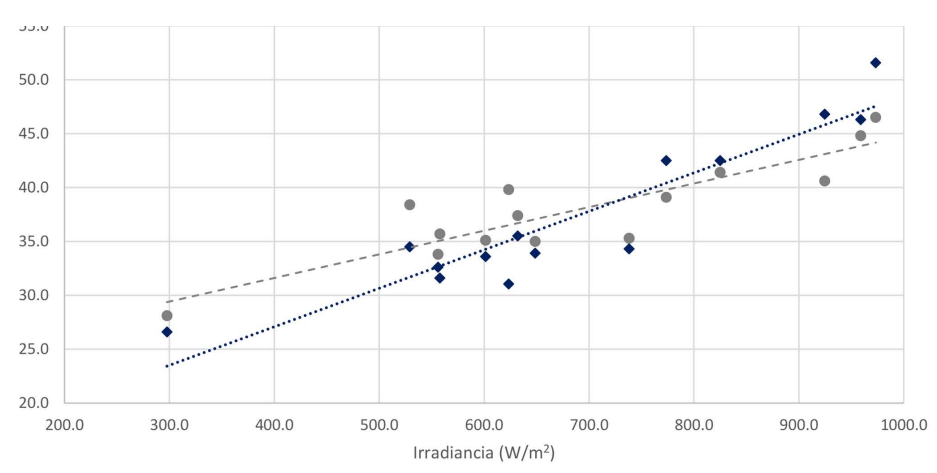

Figura 6. Correlación entre la temperatura del aire y la irradiancia. Fuente: Elaboración de autores. 
Figura 7. Correlación entre la temperatura del aire y la irradiancia. Fuente: Elaboración de autores.
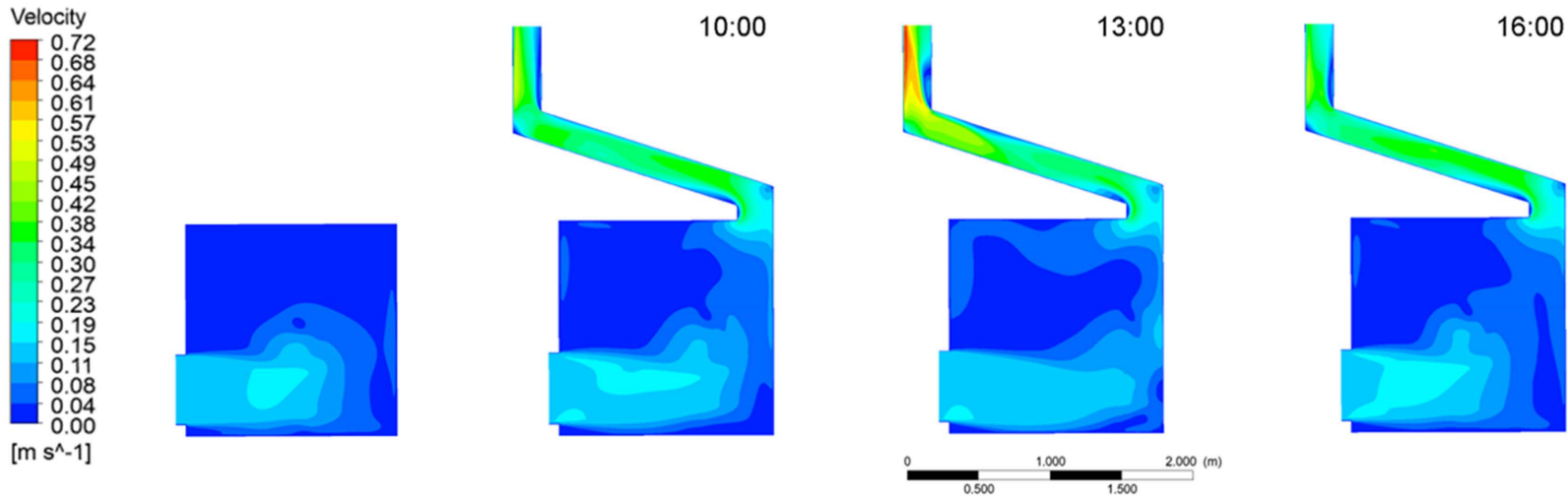

Figura 8. Contornos de velocidad del aire en el plano medio del modelo. Fuente: Elaboración de autores.

Los resultados del comparativo de las velocidades en la salida del tiro entre las mediciones experimentales y las simulaciones bajo diferentes condiciones de irradiancia, se exponen en la Figura 7, donde es notoria la diferencia entre resultados, el coeficiente de correlación entre las mediciones experimentales y los resultados de las simulaciones es de $r=0.23$.

Lo anterior se explica porque el modelo CFD no contempla la influencia del viento que, según lo constatado en las mediciones experimentales, tiene un efecto positivo en la velocidad del aire en la salida del tiro.

La velocidad del aire interior es otra variable considerada al evaluar la ventilación natural, el aumento del movimiento del aire tiene el potencial de cambiar la aceptabilidad térmica a valores de temperatura operativa más altos (Saadatjoo, Mahdavinejad y Zhang, 2018). Para analizar esta variable se obtuvieron contornos de velocidad en el plano medio del modelo. La Figura 8 ilustra los contornos generados por la simulación realizada con los datos experimentales del mes de octubre, mes con mayor cantidad de irradiancia solar disponible dentro del periodo más cálido en la zona de estudio, que corresponde a un promedio de irradiancia mensual de $363 \mathrm{~W} / \mathrm{m}^{2}$ y una temperatura media de $27.6^{\circ} \mathrm{C}$.
Se pueden advertir los cambios en la distribución de la velocidad del aire en tres momentos del día. Tras comparar la información obtenida con una simulación de control (la cámara sin chimenea y sin abertura de salida), se evidencia una mejor circulación del aire a las 13:00 h, cerca del mediodía solar.

\section{CONCLUSIÓN}

Los resultados de esta investigación demuestran que es factible utilizar una extensión vertical en las chimeneas inclinadas para compensar la pérdida de presión ocasionada por la reducción del ángulo de inclinación, y optimizar así las ganancias solares. Una menor inclinación facilitaría la incorporación del sistema a cubiertas con pendientes no mayores al 20\%, como es el caso de la mayoría de las construcciones para uso habitacional en la zona de estudio.

Los datos obtenidos en las simulaciones mostraron una buena concordancia con las mediciones experimentales: el modelo CFD fue capaz de predecir apropiadamente las temperaturas de placa de absorción, con un coeficiente de correlación $r=0.93$ con los resultados experimentales. Las 
temperaturas dentro del colector también mostraron una alta correlación positiva con las medidas experimentales $(r=0.87)$.

Con las simulaciones CFD es posible verificar la importancia que el fenómeno de descarga de calor por flotación natural del aire caliente tiene en este sistema, tanto para los resultados experimentales como para los obtenidos por simulación: se observó, en efecto, una fuerte correlación de la irradiancia con el caudal de aire de la chimenea. El análisis CFD permitió el estudio de los patrones de flujo de aire dentro de la chimenea mediante la visualización de contornos velocidad.

Luego de comparar los resultados CFD con los resultados experimentales se ha llegado a la conclusión de que el modelo computacional describe apropiadamente el comportamiento termo-fluidodinámico de la chimenea solar, utilizando el modelo turbulento k-e RNG complementado con el modelo de radiación Discrete Ordinates.

Las temporadas y horas del día en las que la ventilación natural es más necesaria como estrategia de confort térmico en la zona de estudio, coinciden con las condiciones favorables para proveer mayores tasas de flujo de aire, lo que constata la ventaja del uso de esta configuración de chimenea para la ventilación natural en regiones de baja latitud con clima cálido-húmedo. Se considera que con el uso de chimeneas solares es posible mejorar la ventilación sin gastos económicos y energéticos, puesto que el sistema no necesita consumo de energía convencional y no genera gastos de operación ni mantenimiento.

Las predicciones de la velocidad, temperatura, humedad, concentración de contaminantes, patrones de flujo y tasas de cambio de aire en las edificaciones, son requeridas para el diseño de ambientes interiores saludables y confortables. El enfoque propuesto en esta investigación contribuye al uso práctico del método CFD en el diseño arquitectónico, proporcionando información detallada sobre el modelo numérico utilizado. Los resultados satisfactorios de estas simulaciones permiten concluir que es posible simular y estudiar casos tridimensionales en estado estacionario de una forma asequible y sencilla.

La ventilación natural no solamente es crucial para la conservación de energía y la reducción de las emisiones de carbono, sino también para mejorar el nivel de comodidad y calidad del aire del entorno construido. Recientemente, se ha señalado la importancia de proveer ventilación suficiente en espacios cerrados para promover la renovación de aire interior con aire exterior y reducir la transmisión de enfermedades.

De los análisis efectuados se concluye que la configuración de chimenea solar propuesta tiene un potencial significativo a la hora de incrementar la ventilación natural en locales donde no puede utilizarse la ventilación cruzada, teniendo en cuenta que generalmente las habitaciones de una vivienda se caracterizan por ventilarse por solo una cara. Se recomienda, para estudios posteriores, realizar simulaciones tridimensionales a escala real y analizar la distribución de temperatura en una habitación de una vivienda con la inclusión de puertas, ventanas, cargas térmicas, etc.

\section{AGRADECIMIENTOS}

Este trabajo se desarrolló bajo la tesis "Caracterización de un colector solar de aire con tiro térmico en cubierta para la mejora de la ventilación natural en clima cálido subhúmedo" programa de Doctorado en Diseño Bioclimático, de la División de Ciencias y Artes para el Diseño de la Universidad Autónoma Metropolitana - Se agradece al Centro de Estudios Meteorológicos de la Costa (CEMCO).

\section{REFERENCIAS BIBLIOGRÁFICAS}

Al-Kayiem, H., Sreejaya, K.V. y Gilani, S. (2014). Mathematical analysis of the influence of the chimney height and collector area on the performance of a roof top solar chimney. Energy and Buildings, 68, 305-311. DOI: http://dx.doi.org/10.1016/j. enbuild.2013.09.021

Bassiouny, R. y Korah, N. (2009). Effect of solar chimney inclination angle on space flow pattern and ventilation rate. Energy and Buildings, 41, 190-196. DOI: 10.1016/j.enbuild.2008.08.009

Bouchair, A. (1994). Solar Chimney for Promoting Cooling Ventilation in Southern Algeria. Building Services Engineering Research and Technology, 15, 81-93.

Chen, Z., Bandopadhayay, P., Halldorsson, J., Byrjalsen, C., Heiselberg, P. y Li, Y. (2003). An experimental investigation of a solar chimney model with uniform wall heat flux. Building and Environment, 38, 893-906.

Dhahri, M. y Aouinet, H. (2020). CFD investigation of temperature distribution, air flow pattern and thermal comfort in natural ventilation of building using solar chimney. World Journal of Engineering, 17, 78-86. DOI: https://doi.org/10.1108/WJE-092019-0261

De Buen, O. (2017). El confort en la demanda eléctrica. Recuperado de https://www.energiaadebate.com/blog/2338/

González, L. y Beele, A. (2016). Estudio de caracterización del uso de aire acondicionado en vivienda de interés social. Comisión Nacional para el Uso Eficiente de la Energía. Recuperado de https://www.gob.mx/cms/uploads/attachment/ file/164221/Conuee_2016_Estudio_Caracterizaci_n_Uso_Aire_ Acondicionado_en_Vivienda.pdf

Harris, D. y Helwig, N. (2007). Solar chimney and building ventilation. Applied Energy, 84, 135-146. DOI: 10.1016/j. apenergy.2006.07.001 
Instituto Nacional de Estadística y Geografía [INEGI] (2018). Encuesta Nacional de Consumo de Energéticos en Viviendas Particulares (ENCEVI) 2018. Recuperado de https://www.inegi. org.mx/contenidos/programas/encevi/2018/doc/encevi2018_ presentacion_resultados.pdf

Jianliu, X. y Weihua, L. (2013). Study on solar chimney used for room natural ventilation in Nanjing. Energy and Buildings, 66, 467-469. DOI: http://dx.doi.org/10.1016/j.enbuild.2013.07.036

Khanal, R. y Lei, C. (2011). Numerical investigation of the ventilation performance of a solar chimney. ANZIAM Journal, 52, 899-913. DOI: https://doi.org/10.1016/j.enbuild.2011.03.035

Kong, J., Niu, J. y Lei, C. (2020). A CFD based approach for determining the optimum inclination angle of a roof-top solar chimney for building ventilation. Solar Energy, 198, 555-569. DOI: https://doi.org/10.1016/j.solener.2020.01.017

Lal, S., Kaushik, S. y Bhargav, P. (2013). Solar chimney: A sustainable approach for ventilation and building space conditioning. International Journal of Development and Sustainability, 2(1), 277-297. Recuperado de: https://isdsnet. com/ijds-v2n1-20.pdf

Li, A., Jones, P., Zhao, P. y Wang, L. (2004) Heat transfer and natural ventilation airflow rates from single-sided heated solar chimney for buildings. Journal of Asian Architecture and Building Engineering 3(2): 233-238. DOI: 10.3130/jaabe.3.233

Liping, W. y Angui, L. (2004). A numerical study of vertical solar chimney for enhancing stack ventilation in buildings. En Proceedings of the 21th conference on passive and low energy architecture, Eindhoven, The Netherlands (pp. 1-5). Recuperado de http://alexandria.tue.nl/openaccess/635611/ p1145final.pdf

Mahdavinejad, M., Fakhari, M. y Alipoor, F. (2013). The Study on Optimum Tilt Angle in Solar Chimney as a Mechanical Eco Concept. Frontiers of Engineering Mechanics Research, 2(3), 71-80. Recuperado de https://www.researchgate.net/ publication/256198688_The_Study_on_Optimum_Tilt_Angle_ in_Solar_Chimney_as_a_Mechanical_Eco_Concept

Mathur, J., Bansal, N., Mathur, S., Jain, M. y Anupma. (2006). Experimental investigations on solar chimney for room ventilation. Solar Energy, 80, 927-935. DOI: 10.1016/j. solener.2005.08.008

Neves, L. (2012). Chaminé solar como elemento indutor de ventilação natural em edificações. Tesis doctoral. Universidade Estadual de Campinas. Recuperado de http://repositorio. unicamp.br/jspui/handle/REPOSIP/258774

Rodrigues, N., Frick, J., Bejat, T. y Garrecht, H. (2021). Using CFD to Evaluate Natural Ventilation through a 3D Parametric Modeling Approach. Energies, 14(8). DOI: https:// doi. org/10.3390/en14082197

Saadatjoo, P., Mahdavinejad, M. y Zhang, G. (2018). A study on terraced apartments and their natural ventilation performance in hot and humid regions. Building Simulation, 11, 359-372. DOI: https://doi.org/10.1007/s12273-017-0407-7

Saifi, N., Settou, N., Dokkar, B., Negrou, B. y Chennouf, N. (2012). Experimental study and simulation of airflow in solar chimneys. Energy Procedia, 18, 1289 - 1298. DOI: 10.1016/j. egypro.2012.05.146

Saleem, A., Bady, M., Ookawara, S. y Abdel-Rahman, A. (2016). Achieving standard natural ventilation rate of dwellings in a hotarid climate using solar chimney. Energy and Buildings, 133, 360370. DOI: http://dx.doi.org/10.1016/j.enbuild.2016.10.001

Sánchez, E. (2017). Optimización de la fachada de doble piel acristalada con ventilación natural. Metodología de diseño para el análisis de la eficiencia energética del sistema. Tesis doctoral. Universidad Politécnica de Madrid. Recuperado de https:// oa.upm.es/46745/1/EDURNE_SANCHEZ_SALCEDO.pdf

Shi, L., Zhang, G., Cheng, X., Guo, Y., Wang, J. y Chew, M. (2016). Developing an empirical model for roof solar chimney based on experimental data from various test rigs. Building and Environment, 110, 115-128. DOI: http://dx.doi.org/10.1016/j. buildenv.2016.10.002

Shi, L., Zhang, G., Yang, W., Huang, D., Cheng, X. y Setunge, S. (2018). Determining the influencing factors on the performance of solar chimney in buildings. Renewable and Sustainable Energy Reviews, 88, 223-238. DOI: https://doi.org/10.1016/j. rser.2018.02.033

Yusoff, W., Salleh, E., Adam, N., Sapian, A. y Sulaiman, M. (2010). Enhancement of stack ventilation in hot and humid climate using a combination of roof solar collector and vertical stack. Building and Environment, 45, 2296-2308. DOI: 10.1016/j. buildenv.2010.04.018

Zhai, X., Dai, Y. y Wang, R. (2005). Comparison of heating and natural ventilation in a solar house induced by two roof solar collectors. Applied Thermal Engineering, 25, 741-757. DOI: 10.1016/j.applthermaleng.2004.08.001

Zhang, T., Tan, Y., Yang, H. y Zhang, X. (2016). The application of air layers in building envelopes: A review. Applied Energy, 165, 707-734. DOI: https://doi.org/10.1016/j.apenergy.2015.12.108 